\title{
Review
}

Acta Cytologica 2016;60:344-353

DOI: $10.1159 / 000448510$
Received: May 19, 2016

Accepted after revision: July 18, 2016 Published online: September 3, 2016

\section{Utilization of Flow Cytometry in Pediatric Fine-Needle Aspiration Biopsy Specimens}

\author{
Russell Silowash $^{\mathrm{a}}$ Liron Pantanowitz $^{\mathrm{a}}$ Fiona E. Craig ${ }^{\mathrm{c}}$ Jeffrey P. Simons ${ }^{\mathrm{b}}$ \\ Sara E. Monaco ${ }^{\text {a }}$ \\ ${ }^{a}$ Department of Pathology, University of Pittsburgh Medical Center, and ${ }^{b}$ Department of Pediatric Otolaryngology, \\ Children's Hospital of Pittsburgh of UPMC, Pittsburgh, Pa., and ' Department of Pathology, Mayo Clinic, \\ Phoenix, Ariz., USA
}

\section{Key Words}

Cytology · Cytopathology · Fine-needle aspiration · Flow cytometry $\cdot$ Hematopathology $\cdot$ Lymph node, pediatric

\begin{abstract}
Background: Fine-needle aspiration (FNA) coupled with flow cytometry $(\mathrm{FC})$ can be helpful in suspicious pediatric lymph nodes or masses to exclude a lymphoproliferative disorder. The aim of this study was to analyze FC findings in a series of pediatric FNAs and to correlate abnormal findings with follow-up information. Materials and Methods: All pediatric FNAs performed at a tertiary-care children's hospital over a 46-month period that had FC performed were retrospectively analyzed and correlated with follow-up. Results: A total of 163 FNA procedures were performed in children (age $\leq 21$ years), and 47 (28.8\%) of these cases had FC performed. Specimens were mostly obtained from the head and neck (72.3\% of cases). Nine cases (19.1\%) had abnormal FC findings, including double-negative T cells ( $n=3 ; 33.3 \%$ ), double-positive T cells $(n=3 ; 33.3 \%)$, excess $\lambda$ light chains $(\mathrm{n}=1 ; 11.1 \%)$, weak CD34 positivity $(\mathrm{n}=1 ; 11.1 \%)$, and Tlymphoblastic lymphoma $(n=1 ; 11.1 \%)$. Conclusion: Unusual FC results that are not diagnostic of malignancy can be
\end{abstract}

seen in lymph node FNA in a minority of young patients. In our series, these findings were seen mainly in small populations of $T$ cells and occurred primarily in the setting of reactive lymphoid hyperplasia or ectopic thymic tissue. Cytopathologists performing FNA on children should be aware of these abnormalities and, although they may warrant further investigation and follow-up, they are unlikely to be associated with malignancy.

(c) 2016 S. Karger AG, Basel

\section{Introduction}

Lymphadenopathy is a common finding among the pediatric population, which can cause anxiety for the family and the patient. Enlarged cervical, axillary, and inguinal lymph nodes can be normal throughout early childhood, with palpable lymph nodes being most common between the ages of 3 and 5 years [1]. Herzog [2] documented that $44 \%$ of healthy children and $64 \%$ of sick

The findings in this paper were presented in part at the American Society of Cytopathology (ASC) Annual Meeting in 2013.

\section{KARGER}

E-Mail karger@karger.com www.karger.com/acy
(C) 2016 S. Karger AG, Basel

0001-5547/16/0604-0344\$39.50/0
Correspondence to: Dr. Sara E. Monaco

Department of Pathology, University of Pittsburgh Medical Center 5150 Centre Avenue, POB2, Suite 201

Pittsburgh, PA 15232 (USA)

E-Mail monacose@upmc.edu 
children have palpable lymph nodes during health care visits. Although reactive lymph node hyperplasia is the most common cause of lymphadenopathy in children [3], the persistence of enlarged lymph nodes can often worry parents and patients, which can lead to further investigation [4]. Thus, young patients with persistent lymphadenopathy are frequently referred for a biopsy, given that clinicians do not want to miss a case of malignancy in a young patient and due to the fact that watchful waiting may lead to a delayed diagnosis.

Malignancies such as leukemias and lymphomas are rare overall, but they do comprise a large percent of malignancies in young patients and can present with lymphadenopathy as a prominent feature [1]. Thus, pathological sampling with minimally invasive modalities, such as fine-needle aspiration (FNA), or an excisional biopsy is typically performed when there is persistent lymphadenopathy, clinical symptoms, or radiological imaging findings concerning for malignancy. In order to reduce the number of unnecessary excisional biopsies, Soldes et al. [5] determined that the age of the child, the size of the lymph node $(>2 \mathrm{~cm})$, the location of the lymphadenopathy (e.g. supraclavicular), and whether or not the lymph node was fixed to adjacent tissue were all significant factors to discriminate benign versus malignant adenopathies. However, Celenik et al. [6] found that the size of the lymph node, the laterality (unilateral vs. bilateral), and the presence of B symptoms were not significant indicators of malignancy. Despite these somewhat contradictory findings, it is generally accepted that a thorough evaluation and accurate diagnostic testing are critical to excluding malignancy in worrisome or atypical cases. Hambleton and Hewitt [4] proposed an algorithm for the work-up of lymphadenopathy, which includes excisional biopsy of the node. Although excisional biopsy of a lymph node provides a large amount of tissue, there are risks associated with this surgical procedure, including the risks associated with anesthesia and the risks of a surgical biopsy (e.g. bleeding, infection, and nerve damage). Thus, FNA provides a less invasive, quick, and cost-effective modality for tissue sampling and triage of these lesions for ancillary studies like flow cytometry (FC), which can help to further characterize a patient's lymphadenopathy [3].

FNA has been found to be a sensitive and specific diagnostic tool for differentiating between benign and malignant lymphadenopathies in the pediatric and adolescent population [7]. When coupled with FC, FNA can be an even more powerful diagnostic tool because hematopoietic malignancies can more confidently be ruled in or out, and further subclassification of lymphoid populations can be provided. Combining FNA with FC has been previously shown to be an accurate tool for distinguishing benign processes from malignant ones [8]. The purpose of this study was to retrospectively analyze our experience with FNA from pediatric lymphadenopathy or mass lesions, and to determine the diagnostic utility of FC in this setting.

\section{Methods}

A retrospective review of FNA performed in children (age 21 years or younger) from June 2009 to April 2013 (46 months) at the Children's Hospital of Pittsburgh of UPMC was performed in our pathology laboratory information system (CoPath Plus; Cerner Corporation, North Kansas City, Mo., USA). All FNAs were performed either by a cytopathologist using palpation-guided FNA or by an interventional radiologist using ultrasound-guided FNA, with rapid on-site evaluation for preliminary diagnosis and triage. This project was approved by the University of Pittsburgh's Institutional Review Board.

Before the FNA procedure, information was communicated to the patient and/or parents regarding the process of the procedure itself, as well as its potential risks and benefits. Informed consent was obtained from the parents (in patients under the age of 18 years), and assent was obtained from the patient. To perform an FNA, the mass or lymph node was identified and palpated by the pathologist or visualized using ultrasound imaging by an interventional radiologist. Once the mass was identified, the skin over the mass was cleaned with alcohol. A small (23-27 gauge) needle with or without an attached syringe was inserted into the targeted lesion. While employing suction, a brisk back-and-forth motion was applied to the needle, and a sample was collected. Specimens were initially smeared onto 2 glass slides, and the needle was rinsed into a specimen jar containing $10 \%$ formalin for cell block and/or Roswell Park Memorial Institute medium for FC studies. One slide was air-dried and stained with a Diff-Quik stain. The Diff-Quikstained slide was analyzed on site for adequacy and an initial diagnostic interpretation. The second smear slide was immediately placed in $95 \%$ alcohol fixative and later stained with a Papanicolaou stain. If necessary, additional biopsy passes were obtained in the same manner as described before. Specimens were sent to the FC laboratory for FC immunophenotypic analysis at the request of the clinician or in the event that the specimen had an atypical lymphoid population during the evaluation of the Diff-Quik-stained slide. Once the procedure was concluded, the biopsy site was covered with a bandage.

The FC immunophenotypic analysis was performed using an 8-color BD FACSCanto II flow cytometer (BD Biosciences, San Jose, Calif., USA) with numerous antibodies, as shown in table 1. Plots derived from the flow cytometer were analyzed with BD FACSDiva $^{\mathrm{TM}}$ software (BD Biosciences) and interpreted by a board-certified hematopathologist. The FC findings included a description of any atypical or clonal lymphoid populations, and a report was generated for the cytopathologist to incorporate into the final cytology diagnosis. 


\section{Results}

During the 46-month time frame, 163 total fine-needle procedures were performed on children and young patients. Ninety-seven (60\%) of these procedures were performed by the pathologist. Sixty-four cases (39\%) were performed by the interventional radiologist, and 2 specimens (1\%) were obtained via consult from another health care facility. Forty-seven (28.8\%) specimens from 37 males (aged 10 weeks to 17 years, average age 8.6 years) and 10 females (aged 2-19 years, average age 9.6 years) had tissue collected and submitted for FC immunophenotypic studies. The number of FNA procedures by organ site can be found in table 2. In summary, the vast majority of sites biopsied were from the head-and-neck region, with cervical lymph nodes and masses comprising most of these specimens (34 procedures, $72.3 \%$ ). Salivary glands and associated lymph nodes accounted for 4 $(8.5 \%)$ FNA procedures, while pre- and postauricular lymph nodes/masses accounted for 5 (10.6\%) of the procedures. Two thyroid procedures (4.3\%), 1 supraclavicular lymph node procedure (2.1\%), and 1 axillary lymph node procedure $(2.1 \%)$ also obtained tissue for FC workup.

At the time of the final cytological diagnosis, 38 (80.9\%) of the cases with FC had reactive lymphoid processes with no morphological or FC abnormalities, while 9 (19.1\%) had abnormal FC findings. A summary of the 9 patients can be found in table 3. All 9 patients were males between the ages of 10 weeks and 13 years (average 7.0 years). At the time of the cytomorphological evaluation, 7 of the 9 cases (77.8\%) possessed a heterogeneous lymphoid population, and $2(22.2 \%)$ cases had atypical larger cells suspicious for malignancy. On FC, the 9 cases with abnormal FC results included the presence of double-negative T cells $(n=3 ; 33.3 \%)$, doublepositive T cells $(\mathrm{n}=3 ; 33.3 \%)$, excess $\lambda$ light chains $(\mathrm{n}=$ $1 ; 11.1 \%)$, weak CD34 positivity $(\mathrm{n}=1 ; 11.1 \%)$, and findings compatible with T-lymphoblastic lymphoma ( $\mathrm{n}=$ $1 ; 11.1 \%)$.

In addition to FC, other ancillary studies utilized showed that 2 of the cases $(22.2 \%)$ were compatible with Epstein-Barr virus (EBV) infectious mononucleosis due to EBV in situ hybridization and/or serological studies. One patient (11.1\%) had an acute and granulomatous process with negative special stains and microbial cultures. One case (11.1\%) was suspicious for malignancy (suspicious for Hodgkin lymphoma), and another (11.1\%) was positive for malignancy (T-acute lymphoblastic leukemia/lymphoma).
Table 1. Summary of the antibodies used for FC

\begin{tabular}{lll}
\hline Antibody & Source & Clone \\
\hline CD1a PE & Beckman Coulter & BL6 \\
CD2 V450 & BD Horizon & S5.2 \\
Surface CD3 APC-Alexa & Beckman Coulter & UCHT1 \\
$\quad$ Fluor 750 & & \\
Surface CD3 PE-Cy7 & BD & SK7 \\
Cyto CD3 PerCP-Cy5.5 & BD & SK7 \\
CD4 APC & BD & SK3 \\
CD4 PerCP-Cy5.5 & BD & SK3 \\
CD5 APC & BD & L17F12 \\
CD5 PE-Cy7 & BD & L17F12 \\
CD7 FITC & BD & M-T701 \\
CD7 PE & BD & M-T701 \\
CD8 PE & BD & Leu-2a \\
CD8 V500 & BD Horizon & SK1 \\
CD10 APC & BD & HI10a \\
CD20 V450 & BD Horizon & L27 \\
CD34 APC & BD & 8G12 \\
CD56 APC & BD & NCAM16.2 \\
\hline
\end{tabular}

Table 2. Summary of FNA biopsies submitted for FC

\begin{tabular}{lcc}
\hline Site & Total cases & $\begin{array}{l}\text { Total FNA } \\
\text { performed by } \\
\text { the pathologist }\end{array}$ \\
\hline Neck lymph nodes/masses & $34(72.3)$ & $29(87.9)$ \\
Preauricular lymph nodes/masses & $3(6.4)$ & $3(100)$ \\
Postauricular lymph nodes & $2(4.3)$ & $2(100)$ \\
Submandibular lymph nodes & $2(4.3)$ & $0(0)$ \\
Thyroid & $2(4.3)$ & $0(0)$ \\
Parotid gland & $1(2.1)$ & $0(0)$ \\
Submental lymph node & $1(2.1)$ & $1(100)$ \\
Supraclavicular lymph node & $1(2.1)$ & $1(100)$ \\
Axillary lymph node & $1(2.1)$ & $0(0)$ \\
\hline Total & $47(100)$ & $36(78.3)$ \\
\hline
\end{tabular}

Values are presented as numbers (\%).

\section{Detailed Clinicopathological Features in Cases with Abnormal FC Results}

Of the 9 patients with abnormal FC results, 7 (77.8\%) had a detailed clinical and/or pathological follow-up. Patient number 1 had a history of a neck mass that was somewhat responsive to antibiotic therapy. At the time of intraoperative interpretation, the specimen demonstrated a heterogeneous lymphoid population composed predominantly of small lymphocytes, plasmacytoid cells, and occasional larger, atypical cells worrisome for a pos- 
Table 3. Correlation of clinical, cytological, flow cytometric immunophenotypic studies and follow-up

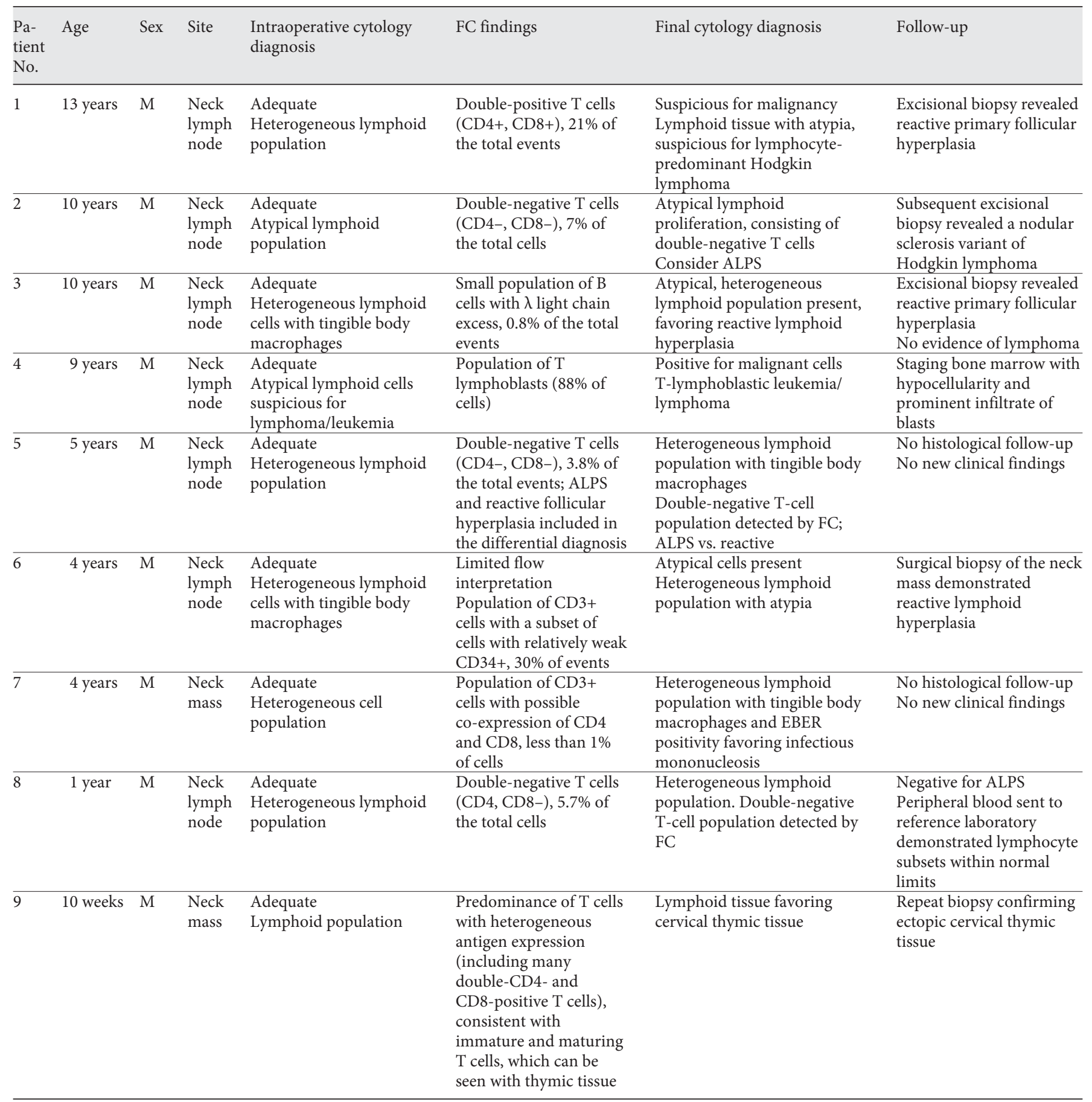


Fig. 1. Benign lymph node with doublepositive T cells detected by FC on preoperative FNA. High-power magnification of the FNA specimen showing mostly small lymphocytes, plasmacytoid cells, and a number of larger, atypical cells. Diff-Quik, high power (a). FC showing a population of CD4- and CD8-coexpressing T cells (double-positive T cells), as seen in the upper right quadrant of the scatterplot (b). Low-power view of the excisional biopsy demonstrating the retained lymph node architecture with expanded follicles. H\&E stain, low power (c). Higher-power view of a reactive lymphoid follicle showing an intact mantle zone and a germinal center with small lymphocytes and occasional tingible body macrophages. H\&E stain, medium power $(\mathbf{d})$.
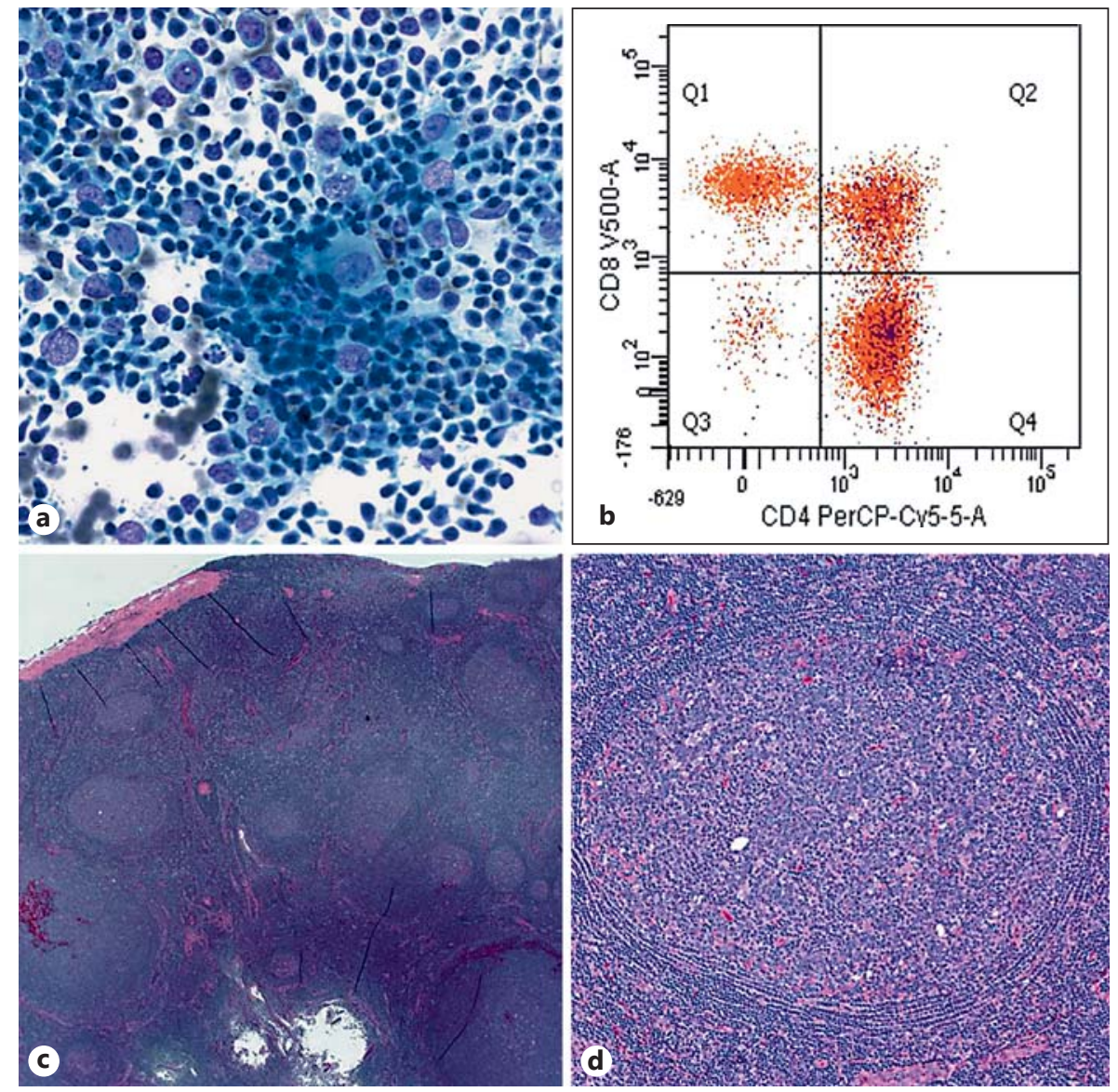

sible Hodgkin lymphoma (fig. 1a). FC studies revealed a population of double-positive T cells (CD4+, CD8+), accounting for $21 \%$ of the total cells (fig. 1b). Upon excisional biopsy, the tissue had findings consistent with reactive lymphoid hyperplasia (fig. 1c, d).

Patient number 2 had a 5-day history of a neck mass for which he was placed on antibiotic therapy. The specimen demonstrated an atypical lymphoid population during intraoperative cytological interpretation, comprised of small-to-medium-sized atypical cells (fig. 2a). FC immunophenotypic studies demonstrated a double-negative (CD4-, CD8-) T-cell population comprising 7\% of the total events (fig. $2 \mathrm{~b}$ ), which prompted a work-up to exclude autoimmune lymphoproliferative syndrome (ALPS). An excisional biopsy was performed, which yielded a diagnosis of Hodgkin lymphoma, nodular sclerosis variant (fig. 2c).

Patient number 3 had a history of an enlarged neck lymph node and tonsillitis. The lymph node demonstrated a heterogeneous lymphoid population at the time of the initial evaluation. FC showed a very small population of B cells with $\lambda$ light chain expression, which was of uncertain significance. A subsequent excisional biopsy demonstrated reactive primary follicular hyperplasia. No autoimmune or immune deficiency was noted on followup.

Patient number 4 had a medical history significant for a large anterior mediastinal mass, diffuse lymphadenopathy, and elevated serum lactate dehydrogenase. A cervical lymph node was sampled by FNA, which demonstrated findings suspicious for a lymphoblastic lymphoma in a rapid on-site evaluation (fig. 3a). Tissue samples analyzed by FC demonstrated a blast population with the following phenotype: $\mathrm{CD} 1 \mathrm{a}+, \mathrm{CD} 2+$, $\mathrm{CD} 7+, \mathrm{CD} 5+, \mathrm{CD} 4+, \mathrm{CD} 8+, \mathrm{CD} 10+, \mathrm{CD} 56-$, cytoplasmic $\mathrm{CD} 3+$, TdT+, surface CD3-, and partial CD34 positivity. These cells accounted for $88 \%$ of the total cell population (fig. $3 \mathrm{~b}, \mathrm{c}$ ). The FC findings, in this case, solidified the final diagnosis of T-lymphoblastic leukemia/ lymphoma and were confirmed via an excisional biopsy (fig. 3d). Subsequent bone marrow involvement was 

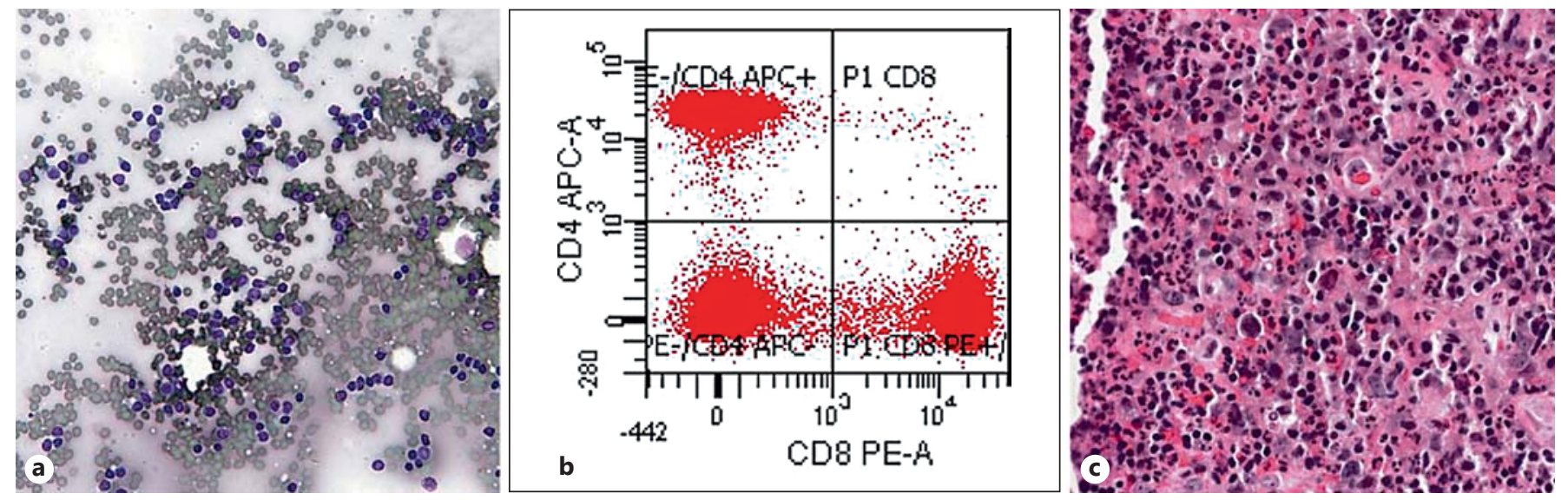

Fig. 2. Classical Hodgkin lymphoma with double-negative T cells detected by FC on preoperative FNA. Low-power view of the FNA specimen demonstrating small-to-medium-sized lymphocytes without Reed-Sternberg cells. Diff-Quik stain, medium power (a). FC of the specimen demonstrating a population of CD4-, CD8-

cells (double-negative T cells) in the left lower quadrant of the plot (b). Intermediate power view of the excisional biopsy demonstrating large Reed-Sternberg cells surrounded by small lymphocytes and eosinophils compatible with classical Hodgkin lymphoma. H\&E stain, intermediate power (c).

Fig. 3. T-lymphoblastic leukemia/lymphoma. High-power view of the FNA specimen showing large monotonous lymphoblasts and atypical mitoses. Diff-Quik stain, high power (a). FC demonstrating a large population of lymphocytes with the immunophenotype $\mathrm{TdT}+, \mathrm{CD} 3+, \mathrm{CD} 4+$, and $\mathrm{CD} 8+$ (b, c). Excisional biopsy at high power showing many monotonous, intermediatesized lymphoid cells with mitoses and apoptotic bodies, imparting a starry sky pattern. These findings are compatible with a T-lymphoblastic leukemia/lymphoma. H\&E stain, high power (d).
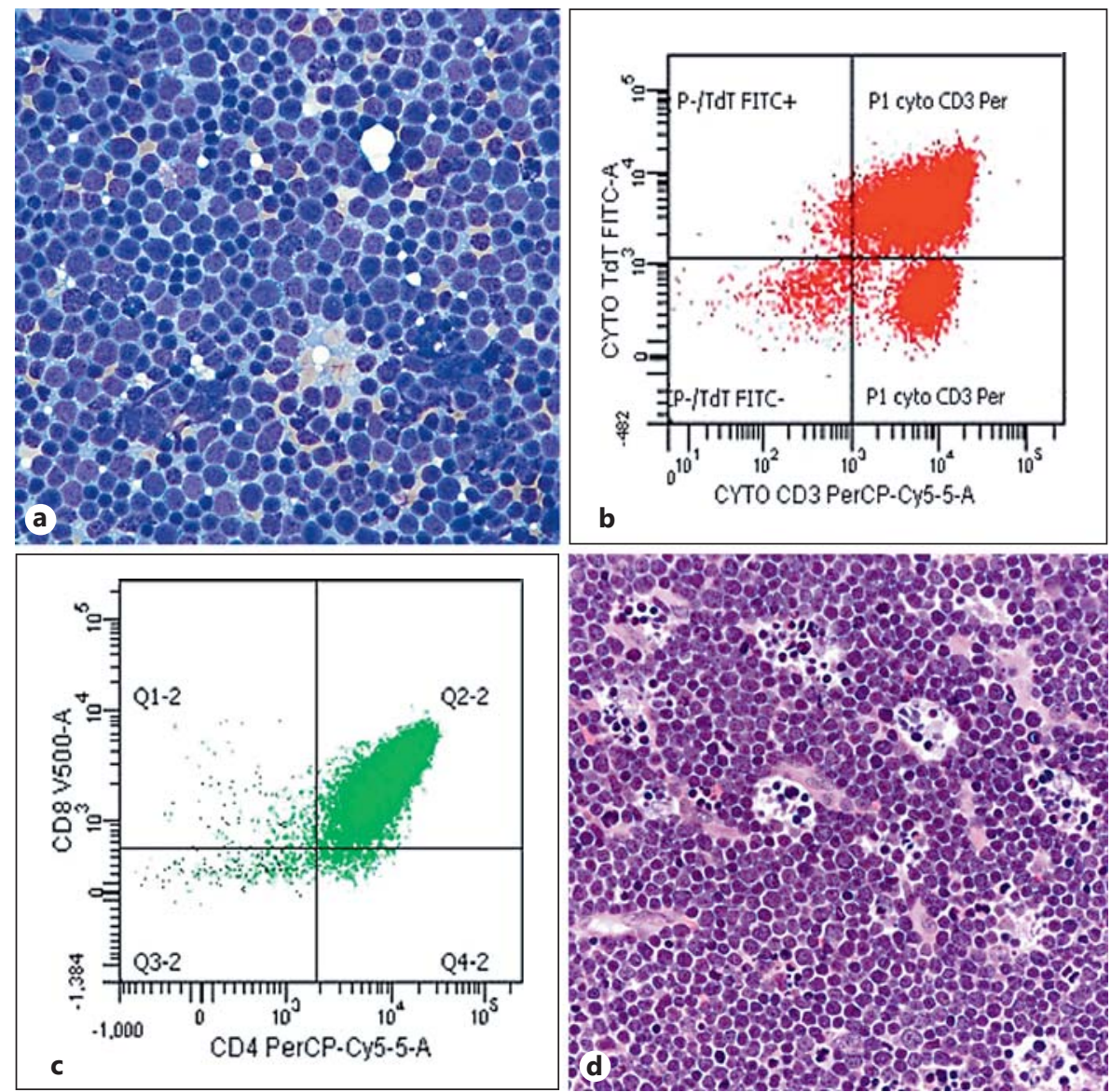
found in this patient during the staging bone marrow biopsy.

Patient number 5 had a history of recurrent tonsillitis and enlarged neck lymph nodes. At the time of intraoperative interpretation, the sample showed a heterogeneous lymphoid population. FC demonstrated a doublenegative T-cell population, which can be seen with ALPS, but the patient was subsequently lost to follow-up.

Patient number 6 had posterior cervical lymphadenopathy and elevated serum lactate dehydrogenase. The initial cytological interpretation showed a heterogeneous population of lymphocytes with tingible body macrophages and occasional larger cells. FC analysis showed a population of CD3-positive cells with a weak CD34 expression in $30 \%$ of the cells. These findings resulted in a final cytological diagnosis of an atypical hematolymphoid population; upon a subsequent surgical excision, the findings were found to be consistent with reactive lymphoid hyperplasia.

Patient number 7 had a prolonged period of lymphadenopathy, an enlarging lymph node unilaterally, and serological studies positive for EBV. The lymph node had a heterogeneous appearance in the initial cytological evaluation. FC findings were significant for a very small population of double-negative $\mathrm{T}$ cells that comprised less than $1 \%$ of all cellular events. The cell block was positive for EBV-encoded RNA by in situ hybridization. There were no follow-up surgical specimens for this patient.

Patient number 8 had a history of an enlarging neck mass for approximately 6 months. The initial interpretation revealed a heterogeneous population of lymphoid cells. A population of double-negative T cells comprising $5.7 \%$ of the total cellular events was discovered by FC; thus, the possibility of ALPS was considered, and blood was sent to a reference lab for evaluation, which excluded the possibility of ALPS as all lymphocyte subsets were found to be within the normal limits.

Finally, patient 9 was the youngest patient, i.e. less than 6 months old, who presented with a neck mass that showed immature and maturing $\mathrm{T}$ cells, including a large population of double-positive T cells with CD4 and CD8, compatible with thymic tissue. The aspirates showed a heterogeneous lymphoid population with mostly small lymphocytes, no conspicuous germinal centers or tingible body macrophages, and scattered epithelioid cells. A follow-up core biopsy confirmed the presence of cervical thymic tissue.

\section{Discussion}

This study investigated the use of FC in pediatric lymphadenopathy at a large tertiary-care medical center with an active, busy pediatric FNA biopsy service. In this setting, approximately $28.8 \%$ of the pediatric FNA utilized FC, making it the most commonly utilized ancillary study for pediatric FNA. This is likely due to the fact that hematopoietic neoplasms are quite common in young patients, comprising $30-45 \%$ of all childhood malignancies $[9,10]$. As in other studies [11-14], most of these FNA were from lesions in the head and neck and were diagnostic of benign/reactive lymphoid hyperplasia with cellular polymorphous lymphocytes, tingible body macrophages, and dendritic cells $[9,10]$. The exact etiology in the majority of cases of reactive lymphoid hyperplasia is unknown $[15,16]$; however, some malignancies such as Hodgkin lymphoma can have a heterogeneous or benign background, which can make the diagnosis challenging [9]. Zardawi et al. [17] found that FNA is an accurate method for detecting hematological malignancies and, when coupled with FC, aspirates can provide the immunophenotype for such malignancies. In our population there was one patient with a T-lymphoblastic lymphoma whose immunophenotype was successfully phenotyped with FC, but overall there was a low incidence of malignancy given that pediatric FNAs in our practice are typically reserved for lesions that are clinically thought to be benign. The information obtained from an FNA with FC can be used to make a definitive diagnosis based on a minimally invasive small biopsy without having to do a more invasive excisional biopsy.

Overall, the most common abnormal finding in pediatric FNA cases sent for FC at our institution involved T-cell populations ( $77.7 \%$ cases sent for FC). One third of the cases had populations of double-negative $\mathrm{T}$ cells detected by FC. Double-negative T cells are those that are $\mathrm{CD} 3$ positive, $\mathrm{CD} 4$ negative, and $\mathrm{CD} 8$ negative. Double-negative $\mathrm{T}$ cells are usually found in the thymus and are precursors to CD4-positive, CD8-positive (double-positive) $\mathrm{T}$ cells and subsequent single-positive (CD4-positive or CD8-positive T cells) during $\alpha \beta \mathrm{T}$-cell development. However, double-negative T cells can also be found in the peripheral blood, the lungs, and the genital tract, as well as in the liver after transplantation or infection. Several studies have described a duality of double-negative $\mathrm{T}$ cells in that they have both a pathogenic and an immunosuppressive role within the human immune system, and they have been seen with some au- 
toimmune disease like systemic lupus erythematosus [18-20]. The presence of a significant double-negative T-cell population can also raise the possibility of ALPS, which presents clinically as splenomegaly, hypergammaglobulinemia, lymphadenopathy, elevated levels of double-negative T cells, and /or autoimmunity. The disease is caused by defective FAS-mediated apoptosis, and it predisposes patients to the development lymphoma, leukemia, and other malignancies [21]. Lim et al. [22] found that patients with ALPS have lymph nodes that usually demonstrate florid follicular hyperplasia with or without a progressive transformation of the germinal centers, polycolonal plasmacytosis, and extensive paracortical proliferation, which could often mimic lymphoma. Testing for ALPS includes a quantitative FC assay performed on whole blood to measure the levels of $3 \mathrm{~T}$ cell subsets, e.g. TCR- $\alpha \beta+$ CD $4-$ CD8 - T cells, CD5+ and CD20+ lymphocytes, and CD5+, HLA-DR+ lymphocytes, which are elevated in patients with ALPS [23]. Of the 3 patients with double-negative $\mathrm{T}$ cells in this study, 1 underwent testing that excluded the diagnosis of ALPS, 1 had an excisional biopsy showing classical Hodgkin lymphoma of the nodular sclerosing subtype, and 1 had benign clinical follow-up.

Another third of the patient samples revealed a population of CD4+, CD8+ (double-positive) T cells. Although double-positive $\mathrm{T}$ cells are a normal part of mature thymocyte development and account for $80 \%$ of intrathymic thymocytes, most of them undergo cell death, but normal mature double-positive T cells can persist in healthy patients [24]. The presence of peripheral doublepositive $\mathrm{T}$ cells has been found in a wide variety of conditions including nodular lymphocyte-predominant Hodgkin lymphoma, classical Hodgkin lymphoma, and progressive transformation of germinal centers [25], as well as autoimmunity [26]. Ortolani et al. [27] demonstrated characteristic FL phenotypes in double-positive T-cell patients across a number of diseases. Of note, patients with EBV-infectious mononucleosis have also been found to have lymphocytes with a dim CD4 and CD8 expression. In our patient with the small population of double-positive $\mathrm{T}$ cells, EBV in situ hybridization studies were positive on the cell block, compatible with the diagnosis of infectious mononucleosis. Nascimbeni et al. [28] showed that double-positive T cells are mature memory cells that respond to viral antigens and vaccine antigens, which may explain the association with EBVrelated infection. One patient with a large population of double-positive $\mathrm{T}$ cells had a few larger cells seen on aspirates, leading to concern for Hodgkin lymphoma.
However, on excision and follow-up the patient was only found to have reactive lymphoid hyperplasia and the mass decreased in size with the administration of antibiotics. Rahemtullah et al. [25] showed that double-positive T-cell populations comprising at least $10 \%$ of the total T-cell population had a $58.3 \%$ sensitivity and a $96.2 \%$ specificity for the diagnosis of nodular lymphocyte-predominant Hodgkin lymphoma. Thus, in the appropriate clinical setting, the detection of double-positive $\mathrm{T}$ cells may warrant excision in cases with cytological atypia to exclude difficult diagnoses such as nodular lymphocytepredominant Hodgkin lymphoma, as in our case. Another etiology of double-positive $T$ cells can be ectopic thymic tissue, which is typically seen in young patients (as in our patient No. 9), and it may provide a benign explanation for a lymphoid neck mass in a young child even in the absence of detectable epithelial cells [29-31]. The key findings in these cases include a mixture of epithelioid cells, which are larger cells with round-to-oval nuclei and more abundant cytoplasm, with small lymphocytes and a relative absence or paucity of plasma cells, which are seen with lymphocytic thyroiditis, in addition to a relative paucity of tingible body macrophages and germinal center cells which are seen in reactive lymphoid hyperplasia [29].

Other FC abnormalities, particularly abnormal B-cell findings with excess light chains or weak CD34 staining, were infrequent and restricted to rare cases, which may be due to the limited cellularity in some cases and nonspecific staining detected by a sensitive test like FC. In addition, clonal B-cell populations have been described in the literature in nonmalignant lymphoid proliferations and may reflect an abnormal response to antigen stimulation, particularly in patients with autoimmune or immunodeficiency syndromes [32-34]. In general, T-cell abnormalities and T-cell lymphoblastic leukemia/lymphoma were more commonly detected and often led to a further clinical evaluation and work-up.

In conclusion, persistent pediatric lymphadenopathy can make parents, children, and clinicians anxious. In addition, these same cases can be challenging for pathologists to evaluate. Although most cases are benign in nature, reactive-appearing processes can show an increase in immunoblastic cells or larger lymphocytes, which may raise concern for more common pediatric malignancies like acute leukemias and Hodgkin lymphomas. Although FC can be helpful to characterize some lymphoproliferative processes, it is often less helpful in children given the relatively rare occurrence of non-Hodgkin lymphoma and the very low risk of a small B-cell lymphoma, which 
is in contrast to the evaluation of lymph node FNAs in adults where FC is frequently utilized. However, occasionally FC is used in cases with atypia to exclude the possibility of a lymphoblastic leukemia/lymphoma or another hematolymphoid malignancy and to rule out thymic tissue with immature and maturing T cells. When FC is submitted, there can be results that are abnormal and of an uncertain significance, and pathologists should be aware of these findings given that clinicians will typically ask for guidance. In this study, we described 9 such cases with abnormal FC that represented less than $20 \%$ of our cases sent for FC. Knowledge of these abnormal results can help when consulting clinicians about abnormal results given that many of these immunophenotypic findings are rare. In addition, from a cytopathologist's perspective, obtaining material for a cell block can help in some of these atypical cases in order to perform additional studies, such as EBV in situ hybridization or immature T-cell markers (e.g. CD1a, TdT), which can help to further clarify the etiology of lymphadenopathy in some cases. Overall, the detection of malignancy is rare in pediatric FNA cases submitted for FC, particularly in most institutions in the USA where FNA is usually reserved for more benign lesions. However, this study showed that, when submitted, FC may be abnormal in a minority of cases and that abnormal T-cell findings predominate over abnormal B-cell findings but are usually benign on follow-up.

\section{Acknowledgements}

We would like to thank the FC laboratory, including Wendy Shallenberger, in the UPMC Division of Hematopathology for their technical assistance with the preparation of this paper.

\section{Disclosure Statement}

All authors report no conflicts of interests with respect to the content of this work.

\section{References}

1 Friedmann AM: Evaluation and management of lymphadenopathy in children. Pediatr Rev 2008;29:53-60.

2 Herzog LW: Prevalence of lymphadenopathy of the head and neck in infants and children. Clin Pediatr (Phila) 1983;22:485-487.

- 3 Monaco SE, Khalbuss WE, Pantanowitz L: Benign non-infectious causes of lymphadenopathy: a review of cytomorphology and differential diagnosis. Diagn Cytopathol 2012; 40:925-938.

4 Hambleton LSJ, Hewitt M: Lymphadenopathy in children and young people. Paediatr Child Health 2015, DOI: http://dx.doi. org/10.1016/j.paed.2015.10.005.

5 Soldes OS, Younger JG, Hirschl RB: Predictors of malignancy in childhood peripheral lymphadenopathy. J Pediatr Surg 1999;34: 1447-1452.

6 Celenk F, et al: Incidence and predictors of malignancy in children with persistent cervical lymphadenopathy. Int J Pediatr Otorhinolaryngol 2013;77:2004-2007.

7 van de Schoot L, Aronson DC, Behrendt H, Bras J: The role of fine-needle aspiration cytology in children with persistent or suspicious lymphadenopathy. J Pediatr Surg 2001; 36:7-11.

8 Liu K, Stern RC, Rogers RT, Dodd LG, Mann KP: Diagnosis of hematopoietic processes by fine-needle aspiration in conjunction with flow cytometry: a review of 127 cases. Diagn Cytopathol 2001;24:1-10.
-9 Steliarova-Foucher E, Stiller C, Kaatsch P, et al: Geographical patterns and time trends of cancer incidence and survival among children and adolescents in Europe since the 1970s (the ACCIS project): an epidemiological study. Lancet 2004;364:2097-2105.

10 Pritchard-Jones K, Kaatsch P, SteliarovaFoucher E, Stiller CA, Coebergh JW: Cancer in children and adolescents in Europe: developments over 20 years and future challenges. Eur J Cancer 2006;42:2183-2190.

-11 Monaco SE, Teot LA: Cytopathology of pediatric malignancies: where are we today with fine-needle aspiration biopsies in pediatric oncology? Cancer Cytopathol 2014;122:322336.

12 Lee DH, et al: Clinical value of fine needle aspiration cytology in pediatric cervical lymphadenopathy patients under 12-years-of-age. Int J Pediatr Otorhinolaryngol 2014;78:7981.

13 Anne S, Teot LA, Mandell DL: Fine needle aspiration biopsy: role in diagnosis of pediatric head and neck masses. Int J Pediatr Otorhinolaryngol 2008;72:1547-1553.

14 Niedzielska G, Kotowski M, Niedzielski A, Dybiec E, Wieczorek P: Cervical lymphadenopathy in children - incidence and diagnostic management. Int J Pediatr Otorhinolaryngol 2007;71:51-56.

15 Pambuccian SE, Bardales RH: Lymph Node Cytopathology, ed 1. New York, Springer, 2011, pp 95-113.
16 Ward E, DeSantis C, Robbins A, Kohler B, Jemal A: Childhood and adolescent cancer statistics 2014. CA Cancer J Clin 2014;64:83103.

17 Zardawi IM, Jain S, Bennett G: Flow-cytometric algorithm on fine-needle aspirates for the clinical workup of patients with lymphadenopathy. Diagn Cytopathol 1998;19:274-278.

18 D'Acquisto F, Crompton T: CD3+CD4CD8- (double negative) T cells: saviours or villains of the immune response? Biochem Pharmacol 2011;82:333-340.

19 Crispin JC, et al: Expanded double negative T cells in patients with systemic lupus erythematosus produce IL-17 and infiltrate the kidneys. J Immunol 2008;181:8761-8766.

20 Voelkl S, Gary R, Mackensen A: Characterization of the immunoregulatory function of human TCR-alphabeta+ CD4- CD8- doublenegative T cells. Eur J Immunol 2011;41:739748 .

21 Teachey DT, Seif AE, Grupp SA: Advances in the management and understanding of autoimmune lymphoproliferative syndrome (ALPS). Br J Haematol 2010;148:205-216.

22 Lim MS, et al: Pathological findings in human autoimmune lymphoproliferative syndrome. Am J Pathol 1998;153:1541-1550.

23 Autoimmune lymphoproliferative profile. 2016. ltd.aruplab.com/Tests/Pub/0095505.

24 Dervovic D, Zuniga-Pflucker JC: Positive selection of T cells, an in vitro view. Semin Immunol 2010;22:276-286. 
25 Rahemtullah A, Reichard KK, Preffer FI, Harris NL, Hasserjian RP: A double-positive CD4+CD8+ T-cell population is commonly found in nodular lymphocyte predominant Hodgkin lymphoma. Am J Clin Pathol 2006; 126:805-814

26 Parel Y, Chizzolini C: CD4+ CD8+ double positive (DP) $\mathrm{T}$ cells in health and disease. Autoimmun Rev 2004;3:215-220.

27 Ortolani C, Forti E, Radin E, Cibin R, Cossarizza A: Cytofluorimetric identification of two populations of double positive (CD4+, CD8+) $\mathrm{T}$ lymphocytes in human peripheral blood. Biochemical Biophys Res Commun 1993;191:601-609.
28 Nascimbeni M, Shin EC, Chiriboga L, Kleiner DE, Rehermann B: Peripheral CD4(+) CD8(+) $\mathrm{T}$ cells are differentiated effector memory cells with antiviral functions. Blood 2004; 104:478-486.

29 Lee YY, Wang WC, Li CF: Aspiration cytology of an ectopic cervical thymoma misinterpreted as a lymphoproliferative lesion of the thyroid: a case report. Oncol Lett 2015;10: $1255-1258$

30 Zeppa P, Varone V, Cozzolino I, Salvatore D, Vetrani A, Palombini L: Fine needle cytology and flow cytometry of ectopic cervical thymoma: a case report. Acta Cytol 2010;54(suppl 5):998-1002.

- 31 Ponder TB, Collins BT, Bee CS, Silverberg $\mathrm{AB}$, Grosso LE, Dunphy CH: Diagnosis of cervical thymoma by fine needle aspiration biopsy with flow cytometry: a case report. Acta Cytol 2002;46:1129-1132.
32 Kussick SJ, Kalnoski M, Braziel RM, Wood BL: Prominent clonal B-cell populations identified by flow cytometry in histologically reactive lymphoid proliferations. Am J Clin Pathol 2004;121:464-472.

33 Bhargava P, Parker JA, Dezube BJ: Pitfalls of diagnosis based on abnormal flow cytometry and [(18)F]fluorodeoxyglucose positron emission tomography. Clin Lymphoma Myeloma 2008;8:117-120

34 Cozzolino I, Nappa S, Picardi M, DeRenzo A, Troncone G, Palombini L, Zeppa P: Clonal B-cell population in a reactive lymph node in acquired immunodeficiency syndrome. Diagn Cytopathol 2009;37:910-914. 